\author{
Stephen M. Klisch \\ Associate Professor \\ Mechanical Engineering Department, \\ California Polytechnic State University, \\ San Luis Obispo, CA 93407 \\ e-mail: sklisch@calpoly.edu
}

\section{A Bimodular Polyconvex Anisotropic Strain Energy Function for Articular Cartilage}

A strain energy function for finite deformations is developed that has the capability to describe the nonlinear, anisotropic, and asymmetric mechanical response that is typical of articular cartilage. In particular, the bimodular feature is employed by including strain energy terms that are only mechanically active when the corresponding fiber directions are in tension. Furthermore, the strain energy function is a polyconvex function of the deformation gradient tensor so that it meets material stability criteria. A novel feature of the model is the use of bimodular and polyconvex "strong interaction terms" for the strain invariants of orthotropic materials. Several regression analyses are performed using a hypothetical experimental dataset that captures the anisotropic and asymmetric behavior of articular cartilage. The results suggest that the main advantage of a model employing the strong interaction terms is to provide the capability for modeling anisotropic and asymmetric Poisson's ratios, as well as axial stress-axial strain responses, in tension and compression for finite deformations. [DOI: 10.1115/1.2486225]

\section{Introduction}

The extracellular solid matrix of articular cartilage contains proteoglycans and a crosslinked collagen network. The proteoglycans are negatively charged molecules that primarily resist compressive loads [1,2] while the collagen network primarily resists tensile and shear loads $[3,4]$. Due in part to its complex molecular structure, articular cartilage typically behaves as an anisotropic material with substantial tension-compression asymmetry [5-10] and likely experiences finite, multi-dimensional strains when subject to typical loads $[11,12]$. In particular, both the Young's modulus and Poisson's ratio ${ }^{1}$ are anisotropic and strain dependent, and can be approximately two orders of magnitude greater in tension than in compression $[9,10,13-19]$. Consequently, the development of accurate finite deformation models of the equilibrium elastic response is challenging.

Bimodular elastic and biphasic models have been developed that can model the asymmetric tensile and compressive mechanical properties for infinitesimal strains $[8,10]$. Those models were based on a general bimodular theory for infinitesimal strains [20] in which the material constants may be discontinuous (or jump) across a surface of discontinuity in strain space, provided that stress continuity conditions are satisfied at the surface. Several exponential models for finite deformations allowing for different mechanical properties in tension and compression have been used for the arterial wall [21] and the annulus fibrosus [22]. However, those models have not employed a general bimodular theory that ensures stress continuity across the surface of discontinuity. Recently, a general bimodular theory employing second-order and exponential stress-strain equations was shown to be capable of modeling the anisotropy and asymmetry in Young's modulus for finite deformations [23]. Those results suggested that, when using the bimodular feature, second-order models might provide a material description as accurate as those provided by exponential models. However, the models studied in Ref. [23] were not ca-

${ }^{1}$ In this paper, the terms "Young's modulus" and "Poisson's ratio" will be used to refer to strain-dependent functions because a finite deformation theory is used.

Contributed by the Bioengineering Division of ASME for publication in the JourNAL OF Biomechanical Engineering. Manuscript received May 1, 2006; final manuscript received September 15, 2006. Review conducted by Clark T. Hung. pable of modeling the anisotropy and asymmetry in Poisson's ratio for finite deformations and were not appropriate for use in computational solutions. $^{2}$

The overall goal of this study is to develop an elastic strain energy function for finite deformations of the articular cartilage solid matrix that meets several criteria. First, it should be capable of modeling the nonlinearity, anisotropy, and asymmetry in Young's modulus and Poisson's ratio. Although the desired accuracy of the stress-strain equation may not be the same for all applications, the level of accuracy sought here is likely to be crucial in continuum growth analysis. ${ }^{3}$ In order to meet this criterion, the bimodular feature is employed. Second, it should satisfy stability criteria so that numerical stability of computational solutions can be expected. In order to meet this criterion, a polyconvex strain energy function is developed; polyconvexity guarantees the existence of local minimizers of the strain energy function when subject to boundary conditions [24] while not sharing the limitations of convexity with respect to the violation of invariance requirements and global uniqueness. Third, it should use a relatively low number of parameters needed to model the desired elastic response, so that the material constants are based on a model that is not over-parameterized ${ }^{4}$ and can be determined from a combination of several common experimental protocols.

In a preliminary study, a bimodular polyconvex strain energy function was developed for articular cartilage based on the strain invariants for an orthotropic material [25]; however, that model, nor the earlier second-order and exponential models [23], were capable of modeling the anisotropy and asymmetry of Poisson's ratio. In that polyconvex model [25], there were no strong interaction or coupling terms for the orthotropic strain invariants. Although recent studies have proposed [26] or used [27] strong interaction terms for orthotropic strain invariants, preliminary studies for this work were not successful in using those terms with the bimodular feature. ${ }^{5}$ Also, those studies have not discussed

\footnotetext{
${ }^{2}$ The second-order model in terms of the first Piola-Kirchhoff stress developed in Ref. [23] was shown to satisfy stability criteria; however, the corresponding Cauchy stress was not.

${ }^{3}$ Applications are presented in the "Discussion."

${ }^{4}$ See the "Discussion" for comments on "over-parameterization" in the context of the nonlinear regression analysis used here.

${ }^{5}$ In particular, strong interaction terms that satisfy the bimodular stress-strain continuity conditions stated in Eq. (7) were not found for an orthotropic material.
} 
possible microstructural causes. An aim of this work is to derive bimodular strong interaction terms that are "simple" enough to allow the experimenter to investigate possible microstructural mechanisms.

A recent development of Ref. [28] used two mechanically equivalent secondary fiber families, in addition to primary fiber families, to represent the phenomena of collagen crosslinking in the annulus fibrosus tissue; that model was capable of producing tensile Poisson's ratios that are an order of magnitude greater than those of our earlier studies $[23,25]$. Since the secondary fiber families introduced in Ref. [28] basically serve as strong interaction or coupling terms for the strain invariants related to the primary fiber families, here it was hypothesized that the introduction of strain invariants generated by secondary fiber families will allow a more accurate description of tensile Poisson's ratios for articular cartilage. In contrast to Ref. [28], this development is incorporated into a bimodular polyconvex strain energy function.

The specific objectives are to: (1) adapt the bimodular theory for finite deformations to the present application; (2) develop a bimodular polyconvex anisotropic strain energy function using primary fibers and strong interaction terms generated by secondary fibers; and (3) compare the predictive capability of models with and without the strong interaction terms using experimental data gathered from the literature. The results suggest that using both the bimodular feature and the strong interaction terms facilitates the accurate description of the anisotropic and asymmetric mechanical properties of articular cartilage in finite deformations.

\section{Methods}

Background. The right Cauchy-Green deformation tensor $\mathbf{C}$ is defined as

$$
\mathbf{C}=\mathbf{F}^{T} \mathbf{F}
$$

where $\mathbf{F}$ is the deformation gradient tensor and the superscript $T$ signifies the transpose operator. The Cauchy, first Piola-Kirchhoff, and second Piola-Kirchhoff stress tensors (denoted as $\mathbf{T}, \mathbf{P}$, and $\mathbf{S}$, respectively) are related by

$$
J \mathbf{T}=\mathbf{P F}^{T}=\mathbf{F S F}^{T}
$$

where $J$ is the determinant of $\mathbf{F}$. The stress constitutive equations for a Green-elastic material may be expressed as

$$
\mathbf{S}=2 \frac{\partial W}{\partial \mathbf{C}}=2 \sum_{i=1}^{n} \frac{\partial W}{\partial I_{i}} \frac{\partial I_{i}}{\partial \mathbf{C}}
$$

where $W=\hat{W}\left\{I_{i}\right\}$ is a scalar strain energy function that depends on a set of invariants $I_{i}$ corresponding to the material symmetry group. The fourth-order elasticity tensor is defined as

$$
\mathbf{C}=\frac{\partial \mathbf{S}}{\partial \mathbf{C}}
$$

Bimodular Elasticity for Finite Deformations. Due to the observed tension-compression asymmetry of the articular cartilage solid matrix, a bimodular theory is used. Earlier models [8,10,23] were based on a bimodular theory [20] in which the material constants may be discontinuous (or jump) across a surface of discontinuity in strain space, provided that stress continuity conditions are satisfied at the surface. The bimodular theory of Ref. [20] was developed in terms of the second Piola-Kirchhoff stress and Lagrange strain tensors. Here, that theory is reformulated to use $\mathbf{C}$ instead of the Lagrange strain tensor.

A scalar valued function of $\mathbf{C}$ identifying a surface of discontinuity in the six-dimensional strain space of $\mathbf{C}$ is defined as

$$
g(\mathbf{C})=0
$$

and is restricted to be a function of the invariants corresponding to the material symmetry group. Different strain energy functions may be specified on either side of a surface of discontinuity; i.e.

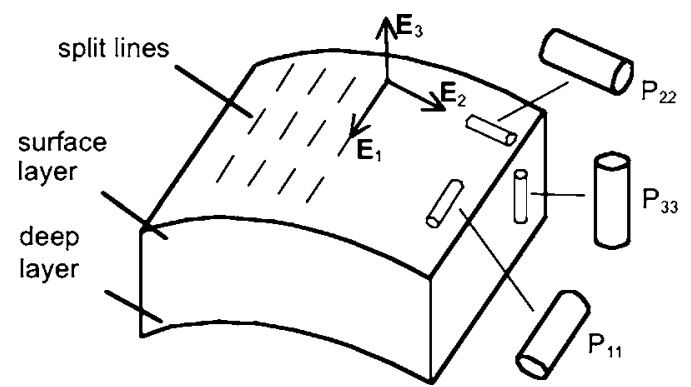

Fig. 1 Schematic of the coordinate system and experimental specimen orientations in relation to anatomical directions. The unit vector $E_{1}$ is parallel to the local split-line direction, the unit vector $E_{3}$ is perpendicular to the articular surface, and the unit vector $E_{2}$ is perpendicular to the split-line direction and parallel to the surface. The cylinders labeled $P_{11}, P_{22}$, and $P_{33}$ represent specimens loaded in tension or compression along the $E_{1}, E_{2}$, and $E_{3}$ directions, respectively.

$$
W=W_{+} \quad \text { if } g(\mathbf{C})>0, \quad W=W_{-} \quad \text { if } g(\mathbf{C})<0
$$

In a similar fashion, different stress and elasticity tensors may be specified on either side of a surface of discontinuity; i.e., as $\left(\mathbf{S}_{+}, \mathbf{S}_{-}, \mathbf{C}_{+}, \mathbf{C}_{-}\right)$

In Ref. [20], a theorem was proved establishing necessary and sufficient conditions for stress continuity across the surface of discontinuity. Introducing a slight modification in Lemma 3.2 of Ref. [20], one obtains the following necessary and sufficient conditions for stress continuity across the surface of discontinuity

$$
\mathbf{S}=\mathbf{S}_{+}=\mathbf{S}_{-}, \quad[[\mathbf{C}]]=\mathbf{C}_{+}-\mathbf{C}_{-}=s(\mathbf{C}) \frac{\partial g}{\partial \mathbf{C}} \otimes \frac{\partial g}{\partial \mathbf{C}}
$$

for all $\mathbf{C}$ that satisfy $g(\mathbf{C})=0$, where $[[\mathbf{C}]]$ represents the jump in the elasticity tensor, $s(\mathbf{C})$ is a scalar valued function of $\mathbf{C}$, and $\otimes$ is the tensor dyadic product.

Structural anisotropy. Spencer [29] proposed a general theory capable of modeling an anisotropic material as a composite material consisting of an isotropic matrix reinforced with fiber families. That theory has been used to develop strain energy functions for cartilaginous tissues [22,28,30-32]. For example, in Ref. [30] two mechanically equivalent fiber families were used to model the annulus fibrosus in finite deformations; in Ref. [28] that model was generalized to include two mechanically equivalent fiber families representing crosslinking phenomena. Also, in Ref. [21] two families of fibers were used to model arterial tissue, but these fiber families were not assumed to be mechanically equivalent because a bimodular feature was used. ${ }^{6}$ Here, secondary fibers are used (as in Ref. [28]) without assuming that the fiber families are mechanically equivalent so that the bimodular feature can be used (as in Ref. [21]).

First, three fiber families are introduced that are parallel to three mutually orthogonal basis vectors $\left(\mathbf{E}_{1}, \mathbf{E}_{2}, \mathbf{E}_{3}\right)$ in a stress-free reference configuration; these will be referred to as "principal fibers." As seen below in Eq. (13), the principal fibers generate strain invariants for orthotropic materials. Structural tensors $\left(\mathbf{M}_{1}, \mathbf{M}_{2}, \mathbf{M}_{3}\right)$ are defined as in the case of an orthotropic material

$$
\mathbf{M}_{1}=\mathbf{E}_{1} \otimes \mathbf{E}_{1}, \quad \mathbf{M}_{2}=\mathbf{E}_{2} \otimes \mathbf{E}_{2}, \quad \mathbf{M}_{3}=\mathbf{E}_{3} \otimes \mathbf{E}_{3}
$$

The unit vectors used to form these structural tensors correspond to the following anatomical directions: $\mathbf{E}_{1}$ is parallel to the local split-line direction, $\mathbf{E}_{3}$ is perpendicular to the articular surface, and $\mathbf{E}_{2}$ is perpendicular to the split-line direction and parallel to the surface (Fig. 1).

Second, two fiber families are introduced in each of the three

\footnotetext{
${ }^{6}$ See the comment below following Eq. (21).
} 


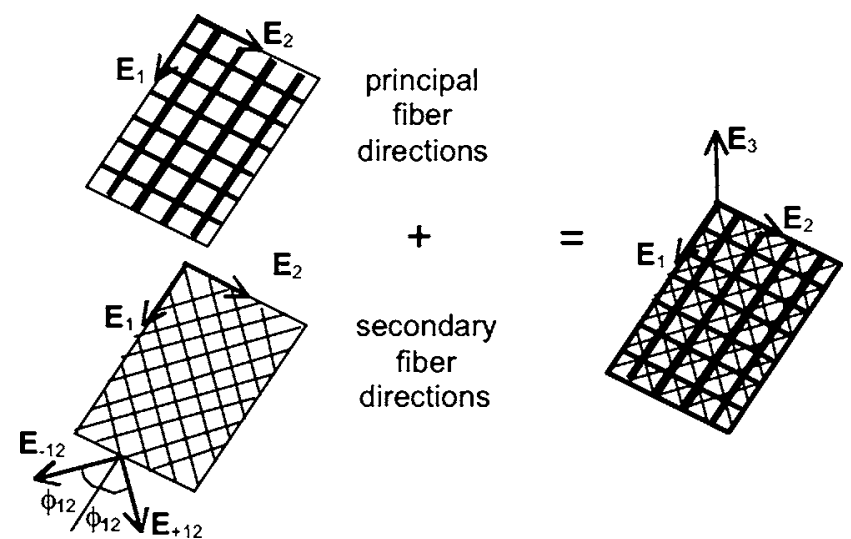

Fig. 2 Schematic of the principal and secondary fiber orientations in relation to anatomical directions in the 1-2 plane. The two principal fiber directions are parallel to the unit vectors $E_{1}$ and $E_{2}$ and the two secondary fiber directions, denoted as $E_{ \pm 12}$, are oriented at angles of $\pm \phi_{12}$ to the $E_{1}$ direction. The weights of the line elements represent the relative strength of the fiber directions as predicted by regression analysis; i.e., the principal fibers along the $E_{1}$ direction are the strongest while the secondary fibers are the weakest.

planes formed by the basis vectors $\left(\mathbf{E}_{1}, \mathbf{E}_{2}, \mathbf{E}_{3}\right)$; these will be referred to as "secondary fibers." As seen below in Eq. (14), the secondary fibers generate strain invariants that represent strong interaction terms between the orthotropic strain invariants. Consider the 1-2 plane, which contains the $\left(\mathbf{E}_{1}, \mathbf{E}_{2}\right)$ unit vectors (Fig. 2 ). The secondary fiber families are defined to lie at angles of $\pm \phi_{12}$ to the $\mathbf{E}_{1}$ direction. These secondary fiber directions are denoted as $\left(\mathbf{E}_{+12}, \mathbf{E}_{-12}\right)$ and are expressed as

$$
\mathbf{E}_{ \pm 12}=\cos \phi_{12} \mathbf{E}_{1} \pm \sin \phi_{12} \mathbf{E}_{2}
$$

Corresponding structural tensors $\left(\mathbf{M}_{+12}, \mathbf{M}_{-12}\right)$ are defined as in Eq. (8)

$$
\begin{aligned}
\mathbf{M}_{ \pm 12}= & \mathbf{E}_{ \pm 12} \otimes \mathbf{E}_{ \pm 12}=\cos ^{2} \phi_{12} \mathbf{E}_{1} \otimes \mathbf{E}_{1}+\sin ^{2} \phi_{12} \mathbf{E}_{1} \\
& \otimes \mathbf{E}_{1} \pm \cos \phi_{12} \sin \phi_{12}\left(\mathbf{E}_{1} \otimes \mathbf{E}_{2}+\mathbf{E}_{2} \otimes \mathbf{E}_{1}\right)
\end{aligned}
$$

The secondary fiber directions introduced in the 1-3 and 2-3 planes are denoted as $\left(\mathbf{E}_{+13}, \mathbf{E}_{-13}\right)$ and $\left(\mathbf{E}_{+23}, \mathbf{E}_{-23}\right)$, respectively, are expressed as

$$
\mathbf{E}_{ \pm 13}=\cos \phi_{13} \mathbf{E}_{1} \pm \sin \phi_{13} \mathbf{E}_{3}, \quad \mathbf{E}_{ \pm 23}=\cos \phi_{23} \mathbf{E}_{2} \pm \sin \phi_{23} \mathbf{E}_{3}
$$

Corresponding structural tensors $\left(\mathbf{M}_{+13}, \mathbf{M}_{-13}, \mathbf{M}_{+23}, \mathbf{M}_{-23}\right)$ are defined as in Eq. (10)

$$
\begin{aligned}
\mathbf{M}_{ \pm 13}= & \cos ^{2} \phi_{13} \mathbf{E}_{1} \otimes \mathbf{E}_{1}+\sin ^{2} \phi_{13} \mathbf{E}_{3} \otimes \mathbf{E}_{3} \\
& \pm \cos \phi_{13} \sin \phi_{13}\left(\mathbf{E}_{1} \otimes \mathbf{E}_{3}+\mathbf{E}_{3} \otimes \mathbf{E}_{1}\right) \\
\mathbf{M}_{ \pm 23}= & \cos ^{2} \phi_{23} \mathbf{E}_{2} \otimes \mathbf{E}_{2}+\sin ^{2} \phi_{23} \mathbf{E}_{3} \otimes \mathbf{E}_{3} \\
& \pm \cos \phi_{23} \sin \phi_{23}\left(\mathbf{E}_{2} \otimes \mathbf{E}_{3}+\mathbf{E}_{3} \otimes \mathbf{E}_{2}\right)
\end{aligned}
$$

Following Ref. [29], the strain energy function $W$ is assumed to be an isotropic function of $\mathbf{C}$ and the nine structural tensors introduced above. In Ref. [33], a procedure is outlined for obtaining minimal lists of irreducible scalar invariants for an arbitrary finite number of symmetric structural tensors; however, that procedure was only employed for up to six symmetric structural tensors. Here, only the decoupled first-order scalar invariants are used in an attempt to obtain a relatively low number of material constants to prevent the model from becoming overparameterized. Invariants associated with the primary fibers include

$$
\left\{\mathbf{M}_{1} \cdot \mathbf{C}, \mathbf{M}_{2} \cdot \mathbf{C}, \mathbf{M}_{3} \cdot \mathbf{C}\right\}=\left\{C_{11}, C_{22}, C_{33}\right\}=\left\{\lambda_{1}^{2}, \lambda_{2}^{2}, \lambda_{3}^{2}\right\}
$$

where $\lambda_{A}^{2}$ represents the square of the stretch of the material line element initially oriented along the principal fiber direction $\mathbf{E}_{A}$. Invariants associated with the secondary fibers include

$$
\begin{aligned}
\left\{\mathbf{M}_{ \pm 12}\right. & \left.\cdot \mathbf{C}, \mathbf{M}_{ \pm 13} \cdot \mathbf{C}, \mathbf{M}_{ \pm 23} \cdot \mathbf{C}\right\} \\
= & \left\{C_{11} \cos ^{2} \phi_{12}+C_{22} \sin ^{2} \phi_{12}\right. \\
& \pm 2 C_{12} \cos \phi_{12} \sin \phi_{12}, C_{11} \cos ^{2} \phi_{13} \\
& +C_{33} \sin ^{2} \phi_{13} \pm 2 C_{13} \cos \phi_{13} \sin \phi_{13}, C_{22} \cos ^{2} \phi_{23} \\
& \left.+C_{33} \sin ^{2} \phi_{23} \pm 2 C_{23} \cos \phi_{23} \sin \phi_{23}\right\} \\
= & \left\{\lambda_{ \pm 12}^{2}, \lambda_{ \pm 13}^{2}, \lambda_{ \pm 23}^{2}\right\}
\end{aligned}
$$

where $\lambda_{+A B}^{2}$ and $\lambda_{-A B}^{2}$ represent the squares of the stretches of the material line elements initially oriented along the secondary fiber directions $\mathbf{E}_{+A B}$ and $\mathbf{E}_{-A B}$, respectively. Note that these invariants represent strong interaction or coupling terms for the orthotropic strain invariants as discussed in Refs. [26,34]; for example, the invariant $\lambda_{+12}^{2}$ is a function of the invariants $C_{11}=\lambda_{1}^{2}$ and $C_{22}$ $=\lambda_{2}^{2}$, thereby coupling these invariants. It is important to emphasize that this approach adopts a lesser level of material symmetry than orthotropy because the invariants in Eq. (14) can easily be shown to not be invariant under transformations due to reflections about three orthogonal planes.

Including the three principal invariants of $\mathbf{C}$, the strain energy function for the model proposed here can be expressed as a function of 12 invariants

$$
W=\hat{W}\left\{\operatorname{tr} \mathbf{C}, \operatorname{tr}(\operatorname{adj} \mathbf{C}), \operatorname{det} \mathbf{C}, \lambda_{1}^{2}, \lambda_{2}^{2}, \lambda_{3}^{2}, \lambda_{ \pm 12}^{2}, \lambda_{ \pm 13}^{2}, \lambda_{ \pm 23}^{2}\right\}
$$

where $\operatorname{tr}$ is the trace operator, adj $\mathbf{C}=(\operatorname{det} \mathbf{C}) \mathbf{C}^{-1}$ is the adjugate of $\mathbf{C}$, and det is the determinant operator.

Bimodular Polyconvex Strain Energy functions. In recent years, polyconvex strain energy functions have been proposed for anisotropic materials $[26,27,34,36]$; discussion of the rationale for using polyconvex strain energy functions is in the "Introduction." A sufficient condition for polyconvexity is as follows [26]: if the strain energy function $W(\mathbf{F})$ satisfies the additive decomposition

$$
W(\mathbf{F})=W_{1}(\mathbf{F})+W_{2}(\operatorname{adj} \mathbf{F})+W_{3}(\operatorname{det} \mathbf{F})
$$

and each of the functions $\left(W_{1}(\mathbf{F}), W_{2}(\operatorname{adj} \mathbf{F}), W_{3}(\operatorname{det} \mathbf{F})\right)$ is a convex function of $(\mathbf{F}, \operatorname{adj} \mathbf{F}, \operatorname{det} \mathbf{F})$, respectively, then $W(\mathbf{F})$ is polyconvex. Furthermore, addition of two or more polyconvex functions results in a polyconvex function.

Here, $W$ is additively decomposed into two terms $W_{O}$ and $W_{\mathrm{BIM}}$ representing nonbimodular and bimodular contributions, respectively. In general, $W_{O}$ can be anisotropic; a general polynomial form is proposed in Ref. [36]. Here, a simple isotropic function is adopted from Ref. [36] for $W_{O}$

$$
W_{O}=\frac{1}{2} \mu\{(\operatorname{tr} \mathbf{C}-3)+(\operatorname{tr}[\operatorname{adj} \mathbf{C}]-3)-3 \ln (\operatorname{det} \mathbf{C})\}
$$

This term is polyconvex if $\mu$ is positive and contributes a stress term as follows [36]

$$
\mathbf{S}_{O}=\mu\left\{\mathbf{I}-(\operatorname{det} \mathbf{C}) \mathbf{C}^{-2}+\left[(\operatorname{det} \mathbf{C}) \operatorname{tr} \mathbf{C}^{-1}-3\right] \mathbf{C}^{-1}\right\}
$$

Then, it is assumed that $W_{\mathrm{BIM}}$ represents the collagen network molecules that account for all of the tissue anisotropy. To model tension-compression asymmetry, it is assumed that all fiber families can only support tensile stresses; consequently, a total of nine surfaces of discontinuity are used

${ }^{7}$ If the secondary fibers are not bimodular and assumed mechanically equivalent, then the symmetry reduces to orthotropy as in $[30,35]$. 


$$
\begin{gathered}
g_{1}=\mathbf{M}_{1} \cdot \mathbf{C}-1=0, \quad g_{2}=\mathbf{M}_{2} \cdot \mathbf{C}-1=0, \quad g_{3}=\mathbf{M}_{3} \cdot \mathbf{C}-1=0 \\
g_{ \pm 12}=\mathbf{M}_{ \pm 12} \cdot \mathbf{C}-1=0, \quad g_{ \pm 13}=\mathbf{M}_{ \pm 13} \cdot \mathbf{C}-1=0 \\
g_{ \pm 23}=\mathbf{M}_{ \pm 23} \cdot \mathbf{C}-1=0
\end{gathered}
$$

For example, the surface $g_{1}=\mathbf{M}_{1} \cdot \mathbf{C}-1=\lambda_{1}^{2}-1=0$ defines a fivedimensional hyperplane that divides the $\mathbf{C}$ space into two halfspaces corresponding to tensile and compressive strains in the principal fiber direction $\mathbf{E}_{1}$. The following bimodular form is used

$$
\begin{aligned}
W_{\mathrm{BIM}}= & \frac{1}{6} \gamma_{1}\left[\lambda_{1}\right]\left(\lambda_{1}^{2}-1\right)^{3}+\frac{1}{6} \gamma_{2}\left[\lambda_{2}\right]\left(\lambda_{2}^{2}-1\right)^{3}+\frac{1}{6} \gamma_{3}\left[\lambda_{3}\right]\left(\lambda_{3}^{2}-1\right)^{3} \\
& +\frac{1}{6} \delta_{ \pm 12}\left[\lambda_{ \pm 12}\right]\left(\lambda_{ \pm 12}^{2}-1\right)^{3}+\frac{1}{6} \delta_{ \pm 13}\left[\lambda_{ \pm 13}\right]\left(\lambda_{ \pm 13}^{2}-1\right)^{3} \\
& +\frac{1}{6} \delta_{ \pm 23}\left[\lambda_{ \pm 23}\right]\left(\lambda_{ \pm 23}^{2}-1\right)^{3}
\end{aligned}
$$

where $\left(\gamma_{1}, \gamma_{2}, \gamma_{3}, \delta_{ \pm 12}, \delta_{ \pm 13}, \delta_{ \pm 23}\right)$ are six material constants that represent bimodular terms via the definitions

$$
\begin{gathered}
\gamma_{1}\left[\lambda_{1}\right]= \begin{cases}\gamma_{1}>0 & \text { if } \lambda_{1}>1 \\
0 & \text { if } \lambda_{1}<1\end{cases} \\
\delta_{+12}\left[\lambda_{+12}\right]=\left\{\begin{array}{ll}
\delta_{+12}>0 & \text { if } \lambda_{+12}>1 \\
0 & \text { if } \lambda_{+12}<1
\end{array},\right. \text { etc }
\end{gathered}
$$

and the angles $\left(\phi_{12}, \phi_{13}, \phi_{23}\right)$ that appear in Eq. (14) can be regarded as three additional material constants. In this general formulation, the two secondary fiber families in any of the three planes will have the same stiffness if both are active (i.e., $\delta_{+12}$ $=\delta_{-12}$ ), but are not assumed to be mechanically equivalent as defined in Ref. [29] because in some shearing deformations one fiber family may be in tension while the other may be in compression. Considering Eq. (21), it is evident that each of the material constants are related to one of the surfaces of discontinuity defined in Eq. (19). For example, the material constant $\gamma_{1}$ defines a strain energy term that can jump across the surface $g_{1}=\mathbf{M}_{1} \cdot \mathbf{C}$ $-1=\lambda_{1}-1=0$.

In the Appendix, the proposed strain-energy function $W_{\mathrm{BIM}}$ is shown to satisfy both the bimodular stress continuity and polyconvexity conditions when the material constants are defined as in Eq. (21). Since preliminary statistical results suggested that the model defined by Eq. (20) was overparameterized given the experimental dataset, ${ }^{8}$ it is further assumed that the material constants associated with the secondary fibers are equal (when active); i.e., $\delta_{ \pm A B}=\delta$. This reduced strain energy function contributes a stress term as

$$
\begin{aligned}
\mathbf{S}_{\mathrm{BIM}}= & \gamma_{1}\left[\lambda_{1}\right]\left(\lambda_{1}^{2}-1\right)^{2} \mathbf{M}_{1}+\gamma_{2}\left[\lambda_{2}\right]\left(\lambda_{2}^{2}-1\right)^{2} \mathbf{M}_{2}+\gamma_{3}\left[\lambda_{3}\right]\left(\lambda_{3}^{2}\right. \\
& -1)^{2} \mathbf{M}_{3}+\delta\left[\lambda_{ \pm 12}\right]\left(\lambda_{ \pm 12}^{2}-1\right)^{2} \mathbf{M}_{ \pm 12}+\delta\left[\lambda_{ \pm 13}\right]\left(\lambda_{ \pm 13}^{2}\right. \\
& -1)^{2} \mathbf{M}_{ \pm 13}+\delta\left[\lambda_{ \pm 23}\right]\left(\lambda_{ \pm 23}^{2}-1\right)^{2} \mathbf{M}_{ \pm 23}
\end{aligned}
$$

so that the stress constitutive equation is defined by Eqs. (18) and (22) as

$$
\mathbf{S}=\mathbf{S}_{O}+\mathbf{S}_{\mathrm{BIM}}
$$

with a total of eight material constants $\left(\mu, \gamma_{1}, \gamma_{2}, \gamma_{3}, \delta, \phi_{12}\right.$, $\left.\phi_{13}, \phi_{23}\right)$.

Experimental Data. A hypothetical experimental dataset was developed that approximates the equilibrium elastic response of the solid matrix of adult human cartilage in the surface region (Tables 1 and 2), assuming homogeneous tissue composition and elastic properties of test specimens that are free of residual stress. In order to construct enough data to prevent the models presented here from being overparameterized, it was necessary to use data

\footnotetext{
${ }^{8}$ These results are summarized in the "Discussion."
}

Table 1 Values of tangent Young's modulus (MPa) in tension at $0 \%$ strain $\left(E_{+0}\right)$ and $16 \%$ strain $\left(E_{+0.16}\right)$ and in compression at $0 \%$ strain $\left(E_{-0}\right)$ and $16 \%$ strain $\left(E_{-0.16}\right)$ in the 1,2 , and 3 directions for the experimental dataset used.

\begin{tabular}{lccc}
\hline \hline & \multicolumn{3}{c}{ Direction } \\
\cline { 2 - 4 } Parameter & 1 & 2 & 3 \\
\hline$E_{+0}$ & 7.8 & 5.9 & 1.2 \\
$E_{+0.16}$ & 42.8 & 26.3 & 9.0 \\
$E_{-0}$ & 0.18 & 0.18 & 0.18 \\
$E_{-0.16}$ & 0.26 & 0.26 & 0.26 \\
\hline \hline
\end{tabular}

from several studies representing different anatomic locations, species, etc.

Based on how mechanical properties were calculated in the studies used here, the first Piola-Kirchhoff stress and Biot strain tensors are used. In particular, the first Piola-Kirchhoff stress normalizes load by original cross-sectional area. Also, the Biot strain tensor has principal strain components (e.g., $E_{11}^{B}, E_{22}^{B}, E_{33}^{B}$ ) that correspond to the definition of the infinitesimal strain tensor $\varepsilon$ (e.g., $E_{11}^{B}=\varepsilon_{11}=\lambda_{1}-1$, etc.). Consequently, Poisson's ratios defined in terms of the Biot strain tensor correspond to the Poisson's ratios defined in terms of $\boldsymbol{\varepsilon}$ used in the studies mentioned below. For example, the Poisson's ratio $\nu_{12}$ is defined here as

$$
\nu_{12}=-E_{22}^{B} / E_{11}^{B}=-\left(\lambda_{2}-1\right) /\left(\lambda_{1}-1\right)=-\varepsilon_{22} / \varepsilon_{11}
$$

The data used corresponds to uniaxial tension (UT) and unconfined compression (UCC) experiments along three directions: 1 $=$ parallel to the split-line; $2=$ perpendicular to the split line and parallel to the surface; and $3=$ perpendicular to the surface (Fig. 1). Exponential functions were used to generate axial (i.e., along the direction of applied loading) stress-axial strain data and linear functions were used to generate transverse strain-axial strain data from $0 \%$ to $20 \%$ strain in $2 \%$ increments. UT axial stress-axial strain data were adopted from Refs. [17,18]. UT Poisson's ratios were assumed based on the results of several studies $[13,15,16,18,37]$. UCC axial stress-axial strain data were adopted from Refs. $[9,14,18]$ and assumed to be the same in all three directions. ' UCC Poisson's ratios were assumed based on the results of Refs. [10,19,38,39].

It is important to note that this hypothetical dataset includes not only substantial anisotropy and asymmetry in the axial stressstrain response (Table 1), but also substantial anisotropy and asymmetry in the Poisson's ratios (Table 2). In particular, the Poisson's ratios in UT can be approximately two orders of magnitude greater than those in UCC, and in both UT and UCC the Poisson's ratios $\nu_{13}$ and $\nu_{23}$ have been measured to be greater than those in other directions; see Refs. [13,37] for UT and Refs. $[10,19]$ for UCC.

Regression Analysis. A simultaneous nonlinear regression algorithm was performed in Mathematica (Wolfram, V5.0) based on an approach developed in Refs. [23,30]. The LevenbergMarquardt method is used to minimize an error term representing the sum of squared differences between theoretical and experimental stress values. Although additional models were studied, ${ }^{10}$ only results of three regression analyses are presented (Table 3). An eight-parameter model (8-PAR) defined by Eqs. (18) and (22) was studied. To provide a comparison with a model that does not use the strong interaction terms, a four-parameter model (4-PAR) was studied, for which the material constant $\delta$ was set equal to zero. For the 8- and 4-PAR models, the assumed Poisson's ratios were used to prescribe the transverse strains for UT and UCC.

\footnotetext{
${ }^{9}$ This limitation is addressed in the "Discussion."

${ }^{10}$ The results of other models are summarized in the Discussion.
} 
Table 2 Numerical values of Poisson's ratios in tension $\left(\nu_{+i j}\right)$ and compression $\left(\nu_{-i j}\right)$ obtained from regression analysis; $i=$ loading direction, $j=$ direction of transverse strain component. The range given corresponds to values at $0 \%$ and $20 \%$ strain. The assumed values are given for comparison in the column labeled Range; tensile values were assumed to be linear functions of strain and the compressive values were assumed to be constant.

\begin{tabular}{|c|c|c|c|c|}
\hline \multirow[b]{2}{*}{ Parameter } & \multirow[b]{2}{*}{ Range } & \multicolumn{3}{|c|}{ Model } \\
\hline & & 8-PAR & 4-PAR & 8-PAR-B \\
\hline$\nu_{+12}$ & $0.5->1.0$ & $0.44->0.83$ & $0.25->0.26$ & $0.79->1.21$ \\
\hline$\nu_{+13}$ & $1.0->2.0$ & $0.75->1.59$ & $0.25->0.26$ & $1.22->1.73$ \\
\hline$\nu_{+21}$ & $0.5->1.0$ & $0.46->0.86$ & $0.25->0.26$ & $0.96->1.54$ \\
\hline$\nu_{+23}$ & $1.0->2.0$ & $0.75->1.59$ & $0.25->0.26$ & $1.08->1.82$ \\
\hline$\nu_{+31}$ & $0.5->1.0$ & $0.32->0.45$ & $0.25->0.26$ & $0.63->0.81$ \\
\hline$\nu_{+32}$ & $0.5 \rightarrow 1.0$ & $0.32->0.45$ & $0.25->0.26$ & $0.71->0.92$ \\
\hline$\nu_{-12}$ & 0.1 & $0.10->0.03$ & $0.11->0.06$ & $0.01->-0.06$ \\
\hline$\nu_{-13}$ & 0.2 & $0.19->0.09$ & $0.60->0.10$ & $0.08->0.04$ \\
\hline$\nu_{-21}$ & 0.1 & $0.09->0.03$ & $0.12->-0.06$ & $0.01->-0.06$ \\
\hline$\nu_{-23}$ & 0.2 & $0.20->0.09$ & $0.21->0.11$ & $0.12->0.12$ \\
\hline$\nu_{-31}$ & 0.1 & $0.11 \rightarrow 0.04$ & $0.13->0.05$ & $0.05->0.02$ \\
\hline$\nu_{-32}$ & 0.1 & $0.13->0.05$ & $0.15->0.06$ & $0.03->0.01$ \\
\hline
\end{tabular}

Then, a composite function representing a total of 18 equations was derived: six axial stress-axial strain equations (three each in UT and UCC), and 12 transverse stress-axial strain equations corresponding to the traction-free boundary conditions (six each in UT and UCC). To provide a comparison with a model that does not explicitly include the 12 traction-free boundary condition equations, an additional regression with the eight-parameter model was performed that included only the six axial stress-axial strain equations obtained after prescribing the transverse strains (8-PAR-B). In all cases, the UCC stress values were weighted by multiplying each stress value by 100 , since the UT stress response is two orders of magnitude greater than the UCC stress response.

After the nonlinear regression analysis was performed, the determined model parameters were used to derive numerical solutions to the UT and UCC boundary-value problems, including theoretical predictions of Poisson's ratios.

\section{Results}

The numerical values for the material constants are presented in Table 3. The nonlinear regression analyses always converged to results consistent with the stability criteria; i.e., $\left(\mu, \gamma_{1}, \gamma_{2}, \gamma_{3}, \delta\right)$ were all positive. The calculated error terms were 0.773 and 0.876 for the 8-PAR and 4-PAR models, respectively, and 0.206 for the 8-PAR-B model. It is important to note that this latter error term cannot be directly compared to the others because fewer equations were used in the nonlinear regression.

Table 3 Numerical values for the material parameters obtained from regression analysis. The constants $\left(\mu, \gamma_{1}, \gamma_{2}, \gamma_{3}, \delta\right)$ are in MPa and the constants $\left(\phi_{12}, \phi_{13}, \phi_{23}\right)$ are in degrees.

\begin{tabular}{lccc}
\hline \hline & \multicolumn{3}{c}{ Model } \\
\cline { 2 - 4 } Parameter & 8-PAR & 4-PAR & 8 -PAR-B \\
\hline$\mu$ & 0.035 & 0.035 & 0.035 \\
$\gamma_{1}$ & 23.13 & 23.88 & 21.92 \\
$\gamma_{2}$ & 14.83 & 15.61 & 1.78 \\
$\gamma_{3}$ & 3.86 & 4.51 & 3.26 \\
$\delta$ & 12.07 & - & 436.8 \\
$\phi_{12}$ & 45 & - & 46 \\
$\phi_{13}$ & 35 & - & 42 \\
$\phi_{23}$ & 35 & - & 40 \\
\hline \hline
\end{tabular}

The predictions of the 8-PAR and 4-PAR models (Figs. 3 and 4), as well as the 8-PAR-B model for axial stresses were qualitatively similar, with one exception. For the 8-PAR-B model that did not explicitly include the traction-free boundary conditions,

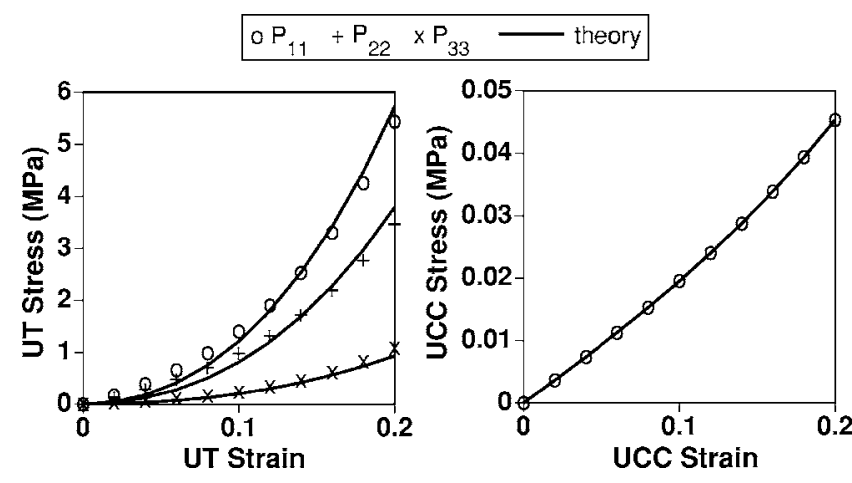

Fig. 3 Predictions of the eight-parameter model (8-PAR) for the uniaxial tension (UT) response in the 1, 2, and 3 directions and the unconfined compression (UCC) response in the $1 \mathrm{di}-$ rection. The theoretical UCC curves in the 2 and 3 directions are within $1 \%$ of the curve shown. UCC stress and strain values, although negative by definition, are plotted as positive numbers.

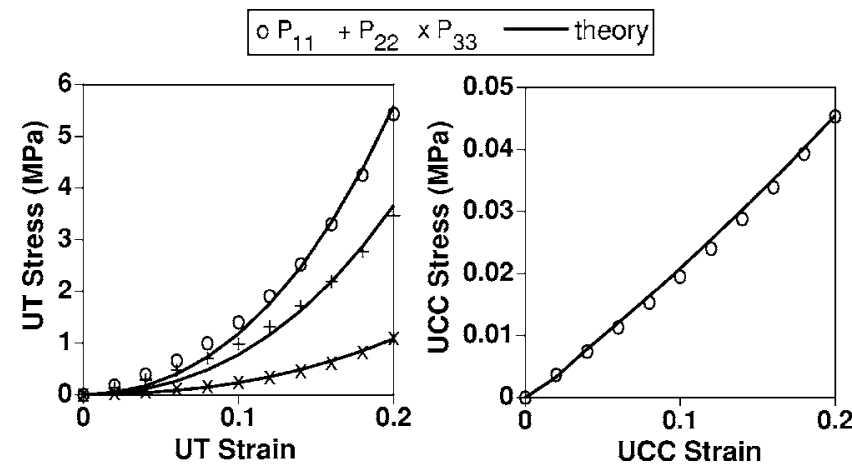

Fig. 4 Predictions of the four-parameter model (4-PAR) for the uniaxial tension (UT) response in the 1, 2, and 3 directions and the unconfined compression (UCC) response in the 1 direction. The theoretical UCC curves in the 2 and 3 directions are within $3 \%$ of the curve shown. UCC stress and strain values, although negative by definition, are plotted as positive numbers. 
the discrepancy between the assumed and theoretical values for UT in the 2 direction was relatively large. Comparing the 8-PAR and 8-PAR-B model results, it is evident that excluding the traction-free boundary condition equations in the regression analysis results in different predicted material constants (Table 3). In particular, the predicted strength $\delta$ of the strong interaction terms was different by an order of magnitude (i.e., 12.07 for the 8-PAR model, 436.8 for the 8-PAR-B model).

In contrast, predictions for Poisson's ratios varied substantially among the models (Table 2). Comparing the 8-PAR and 4-PAR model results, it is evident that including the strong interaction terms facilitates modeling the anisotropic and asymmetric Poisson's ratios, allowing the Poisson's ratios in UT to be 1-2 orders of magnitude greater than those in UCC. Also, the 8-PAR-B model, as compared to the 8-PAR model, provided predicted Poisson's ratios in better and worse agreement with the assumed values in UT and UCC, respectively.

\section{Discussion}

In this paper, a bimodular polyconvex anisotropic strain energy function was developed with the aim of accurately modeling the anisotropic and asymmetric mechanical properties of articular cartilage. The 4-PAR model, based on a bimodular orthotropic material without strong interaction terms, is capable of providing reasonable predictions of the assumed axial stress-axial strain properties in three anatomically relevant directions. However, that model provides a poor description of anisotropic and asymmetric Poisson's ratios. In contrast, the 8-PAR model, which included strong interactions terms for the orthotropic strain invariants, provides a reasonable prediction of anisotropic and asymmetric Poisson's ratios as well as axial stress-axial strain properties. Also, the 8-PAR model was the only one studied that consistently provided theoretical Poisson's ratios in UCC that were positive, in agreement with several studies $[10,17,19,38,39]$. Furthermore, the anisotropic and asymmetric predictions of Poisson's ratios were similar to those measured in several studies; for example, the UCC Poisson's ratio $\nu_{-13}$ was $\sim 3 \times$ greater than $\nu_{-12}$ as compared to experimental values of $5-6 \times[10]$ and $2 \times[19]$, and the UT Poisson's ratio $\nu_{+13}$ was $\sim 2 \times$ greater than $\nu_{+12}$ as compared to an experimental value of $2 \times$ [13]. Although the UCC Poisson's ratios predicted by the 8-PAR model were substantially lower than the assumed values at $20 \%$ strain (Table 2), they were the same order of magnitude as those on the lower end of the reported values $[10,19,38,39]$. Thus, a limitation of the present study is the uncertainty in these predicted UCC Poisson's ratios, as the values assumed were based on experiments using a different tissues source than that assumed for the tensile properties.

The 8-PAR-B model, which did not explicitly include the traction-free boundary conditions in the nonlinear regression analysis, yielded different material constants which resulted in a poor theoretical prediction of UT in the 2 direction. Interestingly, the 8-PAR-B model's theoretical solution for UT stress in the 2 direction based on the assumed Poisson's ratios, as used in the nonlinear regression analysis, was nearly indistinguishable from the experimental curve (result not shown). It appears that the relatively large predicted value of $\delta$ for the 8-PAR-B model, being an order of magnitude greater than the value for the 8-PAR model, appears to magnify the difference between the UT stress calculated using the assumed Poisson's ratios (as used in the nonlinear regression) and using the theoretical Poisson's ratios (as shown in Fig. 5). These results highlight both the importance of including the traction-free boundary condition equations in the regression analysis and checking the complete theoretical solution after the regression analysis is performed.

The approach adopted here is based upon a phenomenological model; a greater understanding of the structure-function relationship for articular cartilage could provide additional insight into degenerative processes and repair strategies. Towards this broader aim, there is insufficient experimental data to completely charac-

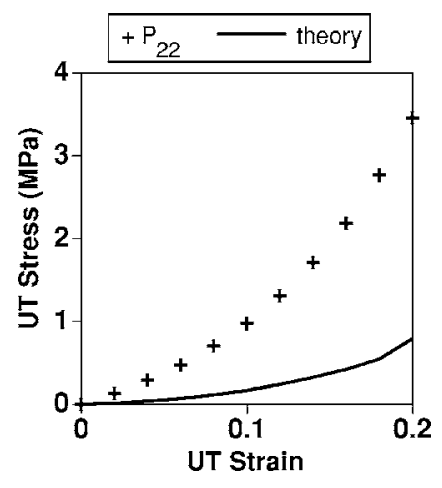

Fig. 5 Prediction of the eight-parameter model that does not include the traction-free boundary condition equations (8PAR-B) for the uniaxial tension (UT) response in the 2 direction. The predictions for the UT response in the 1 and 3 directions and the UCC response in the 1 direction are similar to those of the 8-PAR model shown in Fig. 3.

terize the relationship between the material constants proposed in this study and features of the tissue's microstructure. However, since the present phenomenological model has been derived in accordance with Spencer's theory of fiber-reinforced anisotropy, it does allow us to rationally discuss the possible microstructural interpretations of the material constants. Here, several possible links between the 8-PAR model and microstructural features are presented.

First, the material constant $\mu$ representing the isotropic matrix may be related to the proteoglycan component of the articular cartilage solid matrix; this material constant is a primary determinant of the tissue's compressive stiffness.

Second, the material constants $\left(\gamma_{1}, \gamma_{2}, \gamma_{3}\right)$ associated with the primary fibers may be related to the strength and threedimensional (3D) distribution of collagen fibers. Interestingly, even though the assumed experimental data was proposed to be valid for the superficial region where it is thought that the collagen fibers lie primarily in the split-line direction, the preliminary analysis that neglected the fiber strength in the direction perpendicular to the articular surface (i.e., $\gamma_{3}$ ) did not produce reasonable predictions. This result may suggest a limitation of the present study, as the tensile properties in this direction are not well documented [14].

Third, the material constants $\left(\delta, \phi_{12}, \phi_{13}, \phi_{23}\right)$ associated with the secondary fibers may be related to more than one mechanism. As with the primary fiber strengths $\left(\gamma_{2}, \gamma_{3}\right)$, they may represent fibers that do not lie in the direction of the split-line direction reflecting a 3D distribution of fibers. Also, they may represent crosslinking mechanisms that affect tensile properties, such as collagen-specific crosslinks [40], small proteoglycans [41-43], or bridging fibrils [44]. For example, the latter study [44] proposed an architectural model consisting of cartilage leaves, composed of a fine structure of fibers, that bend towards and become parallel with the surface layer (similar to the classical Benninghoff model [45]). Interestingly, in the surface region those authors state "linking fibrils appeared to bind adjacent collagen leaves to one another" ${ }^{11}$; that observation may provide one microstructural interpretation of fibers (both primary and secondary) other than those aligned with the split-line direction.

Despite the lack of a definitive microstructural interpretation of the material constants used here, the results do suggest that bimodular strong interaction terms for the orthotropic scalar invariants facilitate modeling the asymmetry in Poisson's ratios that have been experimentally measured. From a phenomenological perspective, the secondary fibers in the present model are responsible

\footnotetext{
${ }^{11}$ Quote taken from p. 798 of Ref. [44].
} 
for causing the large lateral contractions in UT that produce Poisson's ratios on the order of 1.0-2.0. In particular, in the UT solution in the 1 direction, the primary fibers in the 2 and 3 directions go into compression; however, the secondary fibers in the 1-2 and 1-3 planes go into tension. Thus, these secondary fibers in the 1-2 and 1-3 planes become mechanically active in a manner that allows them to aid in "contracting" the specimen in the 2 and 3 directions, respectively.

It is possible that other continuum and/or microstructural approaches may yield similar predictions of the anisotropic and asymmetric mechanical response of articular cartilage as adopted here. These approaches may include polyconvex models employing the strong interactions terms proposed in Ref. [34] or used in Ref. [27,36] for orthotropic materials, or anisotropic models based on a 3D distribution of fibers that extend the 2D approach of Ref. [46] such as the recent study of Ref. [47]. However, this study does appear to be the first to develop a polyconvex model that can provide a reasonable prediction for the stresses and Poisson's ratios measured for articular cartilage in UT and UCC in multiple directions.

A secondary aim of this paper was to obtain a relatively simple model using a minimum number of parameters needed to model the desired response. Using a minimum number of parameters facilitates parameter estimation from experimental datasets. In the models presented here, parameter estimates were insensitive to initial values used, as required by the nonlinear regression analysis. As mentioned earlier, many models were studied in addition to those presented in the "Results" section. For example, a tenparameter model was based on the bimodular strain energy function Eq. (20) with different strengths (i.e., $\delta_{ \pm 12} \neq \delta_{ \pm 13} \neq \delta_{ \pm 23}$ ) for the strong interaction terms. Although that model resulted in a lower error (0.721) than the 8-PAR model, the asymptotic correlation matrix and confidence interval statistics suggested that the ten-parameter model was over-parameterized. That conclusion is consistent with the observation that the parameters estimated for that ten-parameter model did depend on initial values, due to overparameterization. Additional models derived as reduced forms of the 8-PAR model were studied; however, none of these models were able to model the anisotropic and asymmetric Poisson's ratios. For example, when the primary fiber strength $\gamma_{3}$ was neglected, the secondary fiber angles $\phi_{12}$ and $\phi_{13}$ converged to values close to $0 \mathrm{deg}$ in the regression, producing predictions very similar to those of the 4-PAR model.

There are limitations related to the assumed experimental dataset. First, the data used did not correspond to a complete set of UT and UCC experiments for a specific source of articular cartilage (i.e., anatomic site, species, age, etc.). However, it did describe a highly anisotropic and asymmetric mechanical response typical of cartilage. Second, the experimental dataset used assumed an isotropic UCC response. In preliminary studies that assumed an anisotropic UCC response, many anisotropic polyconvex strain energy functions generalizing $W_{o}$ were used based on Ref. [36]. Although that formulation can model substantial UCC anisotropy, it introduced additional material constants that rendered the model over-parameterized given the assumed experimental data. Furthermore, there is little data with respect to the anisotropic UCC properties at large deformations. In a recent study [25], we have proposed that a model employing a bimodular anisotropic collagen network (using methods developed here) that restrains an isotropic proteoglycan matrix can explain several anisotropic UCC features observed in young bovine cartilage $[10,17,19]$. In order to address these limitations, we have recently developed experimental protocols to measure confined compression, unconfined compression, and torsion properties in an anisotropic manner at large deformations [19].

It is important to note that the desired accuracy of the stress constitutive equation depends on the application that it is being used for. For example, there is uncertainty regarding the accuracy needed to predict areas of peak stresses and, consequently, regions of failure for articular cartilage of in vivo joints. One study that modeled joint contact using an idealized geometry found that a transversely isotropic model, as compared to an isotropic one, better predicts locations of peak stress that agree with injury location following impact [11]. In contrast, another study using experimental contact pressure measurements and finite-element analysis simulation using computed tomography-generated mesh geometry [48] found that predicted areas of maximum contact pressure may be more sensitive to surface mesh topology.

The primary motivation for the present work was to obtain accurate stress constitutive equations that are needed to conduct robust validation tests of the cartilage growth mixture models that model proteoglycan and collagen growth at different rates [49-54]. If these growth models can be validated for specific in vitro protocols, then it may be possible to accurately predict $3 \mathrm{D}$ geometry changes of graft tissue or tissue engineered constructs that are needed to repair a specific site. Indeed, difficulties associated with one current clinical repair strategy, osteochondral graft implantation, include the construction of a smooth convex joint surface and mismatch between donor and repair site thickness [55]. Current efforts are aimed at describing 3D geometry changes for explants grown in vitro. In order to accurately model, or predict, how thickness and diameter of a cylindrical implant would change during growth, one needs accurate anisotropic stress constitutive equations for tension and compression states. Indeed, current (unpublished) studies are finding substantially different predictions of 3D geometry of constructs grown in vitro using cartilage growth models employing different stress constitutive equations; the results depend on the degree of anisotropy assumed in the constitutive model.

\section{Acknowledgment}

This material is based upon work supported by the National Science Foundation under Grant No. 0245709, the Office of Naval Research (Department of Navy), and the Donald E. Bently Center for Engineering Innovation.

\section{Appendix: Proof of Continuity and Stability Conditions}

Here, it is shown that the strain energy function $W_{\text {BIM }}$ defined in Eq. (20) satisfies the bimodular stress continuity and polyconvexity conditions provided that the restrictions in Eq. (21) are met. It suffices to prove these conditions in a general case; any term in Eq. (20) and its associated surface of discontinuity can be stated in general form as

$$
W_{a}=\frac{1}{6} \gamma_{a}\left(\lambda_{a}^{2}-1\right)^{3}=\frac{1}{6} \gamma_{a}(\mathbf{C} \cdot \mathbf{A}-1)^{3}, \quad g_{a}=\lambda_{a}^{2}-1=\mathbf{C} \cdot \mathbf{A}-1
$$

where $\mathbf{A}=\mathbf{a} \otimes \mathbf{a}$ is a structural tensor defined by a fiber direction $\mathbf{a}$. It is convenient to express Eq. (A1) using indicial notation

$$
W_{a}=\frac{1}{6} \gamma_{a}\left(C_{M N} A_{M N}-1\right)^{3}, \quad g_{a}=C_{M N} A_{M N}-1
$$

Bimodular Stress Continuity Condition. The terms in the stress and elasticity tensors derived from Eq. (A2) are calculated as $S_{A B}=2 \partial W / \partial C_{A B}$ and $\mathbf{C}_{A B C D}=\partial S_{A B} / \partial C_{C D}$, respectively, and are highlighted as follows

$$
\begin{gathered}
S_{A B}=\ldots+\gamma_{a}\left(C_{M N} A_{M N}-1\right)^{2} A_{A B}+\ldots \\
\mathbf{C}_{A B C D}=\ldots+2 \gamma_{a}\left(C_{M N} A_{M N}-1\right) A_{A B} A_{C D}+\ldots
\end{gathered}
$$

Considering the surface of discontinuity defined by $C_{M N} A_{M N}-1$ $=0$, it is seen that $S_{A B}=0$ and $\mathbf{C}_{A B C D}=0$ on this surface. Thus, both the stress and elasticity tensors are continuous at the surface; i.e., $\mathbf{S}=\mathbf{S}_{+}=\mathbf{S}_{-}$and $[[\mathbf{C}]]=\mathbf{C}_{+}-\mathbf{C}_{-}=0$. Consequently, the bimodular stress continuity conditions Eq. (7) are satisfied with the choice of $s(\mathbf{C})=0$.

Polyconvexity Condition. Considering the sufficient conditions for polyconvexity stated in Eq. (16), it suffices to show that 
the strain energy term $W_{a}$ defined in Eqs. (A1) and (A2) is a convex function of $\mathbf{F}$. In indicial notation, the convexity condition requires

$$
\frac{\partial^{2} W_{a}}{\partial F_{i A} \partial F_{j B}} H_{i A} H_{j B}>0 \quad \text { for all } H_{k C} \neq F_{k C}, \quad H_{k C} \neq 0
$$

where $W_{a}$ can be written in terms of $\mathbf{F}$ as

$$
W_{a}=\frac{1}{6} \gamma_{a}\left(F_{k M} F_{k N} A_{M N}-1\right)^{3}
$$

A straightforward differentiation in indicial notation leads to

$$
\begin{aligned}
\frac{\partial^{2} W_{a}}{\partial F_{i A} \partial F_{j B}}= & \gamma_{a}\left(F_{k M} F_{k N} A_{M N}-1\right)^{2} \delta_{i j} A_{A B} \\
& +4 \gamma_{a}\left(F_{k M} F_{k N} A_{M N}-1\right) F_{i P} A_{A P} F_{j Q} A_{Q B}
\end{aligned}
$$

Consequently, one obtains

$$
\begin{aligned}
\frac{\partial^{2} W_{a}}{\partial F_{i A} \partial F_{j B}} H_{i A} H_{j B}= & \gamma_{a}\left(F_{k M} F_{k N} A_{M N}-1\right)^{2} H_{i A} A_{A B} H_{i B} \\
& +4 \gamma_{a}\left(F_{k M} F_{k N} A_{M N}-1\right) F_{i P} A_{A P} F_{j Q} A_{Q B} H_{i A} H_{j B}
\end{aligned}
$$

This result can be expressed in direct notation in terms of the fiber direction a defined by $\mathbf{A}=\mathbf{a} \otimes \mathbf{a}$ and the fiber stretch $\lambda_{a}$ as

$$
\begin{aligned}
\frac{\partial^{2} W_{a}}{\partial \mathbf{F} \partial \mathbf{F}} \mathbf{H} \cdot \mathbf{H}= & \gamma_{a}\left(\lambda_{a}^{2}-1\right)^{2}(\mathbf{H a}) \cdot(\mathbf{H a}) \\
& +4 \gamma_{a}\left(\lambda_{a}^{2}-1\right)[(\mathbf{F a}) \cdot \mathbf{H}] \cdot[(\mathbf{F a}) \cdot \mathbf{H}]
\end{aligned}
$$

Since the scalar products $(\mathbf{H a}) \cdot(\mathbf{H a})$ and $[(\mathbf{F a}) \cdot \mathbf{H}] \cdot[(\mathbf{F a}) \cdot \mathbf{H}]$ are always positive, a necessary and sufficient condition for Eq. (A8) to be positive is that $\gamma_{a}$ be positive when $\lambda_{a}>1$ and equal to zero when $\lambda_{a}<1$, as defined in Eq. (21).

In conclusion, the bimodular stress continuity condition allows the material constant $\gamma_{a}$ to jump across the surface of discontinuity, while the polyconvexity condition further requires that $\gamma_{a}$ be a positive constant on the tensile side and equal to zero on the compressive side.

\section{References}

[1] Lai, W. M., Hou, J. S., and Mow, V. C., 1991, "A Triphasic Theory for the Swelling and Deformation Behaviors of Articular Cartilage," J. Biomech. Eng., 113, pp. 245-258.

[2] Basser, P. J., Schneiderman, R., Bank, R. A., Wachtel, E., and Maroudas, A., 1998, "Mechanical Properties of the Collagen Network in Human Articular Cartilage as Measured by Osmotic Stress Technique," Arch. Biochem. Biophys., 351, pp. 207-219.

[3] Venn, M. F., and Maroudas, A., 1977, "Chemical Composition and Swelling of Normal and Osteoarthritic Femoral Head Cartilage. I. Chemical Composition," Ann. Rheum. Dis., 36, pp. 121-129.

[4] Mow, V. C., and Ratcliffe, A., 1997, "Structure and Function of Articular Cartilage and Meniscus," Basic Orthopaedic Biomechanics, V. C. Mow and W. C. Hayes, eds., Raven Press, New York, pp. 113-178.

[5] Woo, S. L.-Y., Akeson, W. H., and Jemmott, G. F., 1976, "Measurements of Nonhomogeneous Directional Mechanical Properties of Articular Cartilage in Tension,” J. Biomech., 9, pp. 785-791.

[6] Woo, S. L.-Y., Lubock, P., Gomez, M. A., Jemmott, G. F., Kuei, S. C., and Akeson, W. H., 1979, "Large Deformation Nonhomogeneous and Directional Properties of Articular Cartilage in Uniaxial Tension," J. Biomech., 12, pp. 437-446.

[7] Akizuki, S., Mow, V. C., Muller, F., Pita, J. C., Howell, D. S., and Manicourt, D. H., 1986, "Tensile Properties of Human Knee Joint Cartilage: I. Influence of Ionic Conditions, Weight Bearing, and Fibrillation on the Tensile Modulus," J. Orthop. Res., 4, pp. 379-392.

[8] Soltz, M. A., and Ateshian, G. A., 2000, "A Conewise Linear Elasticity Mixture Model for the Analysis of Tension-Compression Nonlinearity in Articular Cartilage," J. Biomech. Eng., 122, pp. 576-586.

[9] Laasanen, M., Toyras, J., Korhonen, R., Rieppo, J., Saarakkala, S., Nieminen, M., Hirvonen, J., and Jurvelin, J. S., 2003, "Biomechanical Properties of Knee Articular Cartilage," Biorheology, 40, pp. 133-140.

[10] Wang, C. C., Chahine, N. O., Hung, C. T., and Ateshian, G. A., 2003, "Optical Determination of Anisotropic Material Properties of Bovine Articular Cartilage in Compression," J. Biomech., 36(3), pp. 339-353.

[11] Donzelli, P. S., Spilker, R. L., Ateshian, G. A., and Mow, V. C., 1999, "Contact Analysis of Biphasic Transversely Isotropic Cartilage Layers and Correla- tions with Tissue Failure," J. Biomech., 32(10), pp. 1037-1047.

[12] Krishnan, R., Park, S., Eckstein, F., and Ateshian, G. A., 2003, "Inhomogeneous Cartilage Properties Enhance Superficial Interstitial Fluid Support and Frictional Properties, but do not Provide a Homogeneous State of Stress," J. Biomech. Eng., 125(5), pp. 569-577.

[13] Chang, D. G., Lottman, L. M., Chen, A. C., Schinagl, R. M., Albrecht, D. R., Pedowitz, R. A., Brossman, J., Frank, L. R., and Sah, R. L., 1999, "The Depth-Dependent, Multi-axial Properties of Aged Human Patellar Cartilage in Tension," Trans. Annu. Meet. - Orthop. Res. Soc., 24, p. 644.

[14] Korhonen, R. K., Toyras, J., Nieminen, M. T., Rieppo, J., Hirvonen, J., Helminen, H. J., and Jurvelin, J. S., 2001, "Effect of Ionic Environment on the Compression-Tension Nonlinearity of Articular Cartilage in the Direction Perpendicular to Articular Surface," Trans. Annu. Meet. - Orthop. Res. Soc., 26, p. 439.

[15] Elliot, D. M., Narmoneva, D. A., and Setton, L. A., 2002, "Direct Measurement of the Poisson's Ratio of Human Patella Cartilage in Tension," J. Biomech. Eng., 124, pp. 223-228.

[16] Charlebois, M., McKee, M. D., and Buschmann, M. D., 2004, "Nonlinear Tensile Properties of Bovine Articular Cartilage and Their Variation with Age and Depth," J. Biomech. Eng., 126, pp. 129-137.

[17] Chahine, N. O., Wang, C. C., Hung, C. T., and Ateshian, G. A., 2004, “Anisotropic Strain-Dependent Material Properties of Bovine Articular Cartilage in the Transitional Range From Tension to Compression," J. Biomech., 37, pp. $1251-1261$.

[18] Huang, C. Y., Stankiewicz, A., Ateshian, G. A., and Mow, V. C., 2005, “Anisotropy, Inhomogeneity, and Tension-Compression Nonlinearity of Human Glenohumeral Cartilage in Finite Deformation," J. Biomech., 38(4), pp. 799_ 809.

[19] Ficklin, T., Thomas, G., Chen, A., Sah, R., Davol, A., and Klisch, S., 2006 "Development of an Experimental Protocol to Measure Anisotropic Material Properties of Bovine Articular Cartilage," Proceedings Summer Bioengineering Conference, ASME, Amelia Island, FL.

[20] Curnier, A., He, Q. C., and Zysset, P., 1995, "Conewise Linear Elastic Materials," J. Elast., 37, pp. 1-38.

[21] Holzapfel, G. A., Gasser, T. C., and Ogden, R. W., 2004, "Comparison of a Multi-layer Structural Model for Arterial Walls with a Fung-Type Model, and Issues of Material Stability," J. Biomech. Eng., 126(2), pp. 264-275.

[22] Baer, A. E., Laursen, T. A., Guilak, F., and Setton, L. A., 2004, "The Micromechanical Environment of Intervertebral Disc Cells Determined by a Finite Deformation, Anisotropic, and Biphasic Finite Element Model,” J. Biomech. Eng., 125, pp. 1-11.

[23] Klisch, S. M., 2006, "A Bimodular Theory for Finite Deformations: Comparison of Orthotropic Second-order and Exponential Stress Constitutive Equations for Articular Cartilage," Biomech. Model. Mechanobiol., 5(2-3), pp. $90-101$

[24] Ball, J. M., 1977, "Convexity Conditions and Existence Theorems in Nonlinear Elasticity," Arch. Ration. Mech. Anal., 63, pp. 337-403.

[25] Klisch, S. M., Sah, R. L., and Davol, A., 2006, "Bimodular-OrthotropicPolyconvex Strain Energy Functions for the Collagen-Proteoglycan Solid Matrix of Articular Cartilage," Proceedings Summer Bioengineering Conference, ASME, Amelia Island, FL.

[26] Schroder, J., and Neff, P., 2003, "Invariant Formulation of Hyperelastic Transverse Isotropy Based on Polyconvex Free Energy Functions," Int. J. Solids Struct., 40, pp. 401-445.

[27] Itskov, M., Ehret, A. E., and Mavrilas, D., 2006, "A Polyconvex Anisotropic Strain-Energy Function for Soft Collagenous Tissues," Biomech. Model. Mechanobiol., 5(1), pp. 17-26.

[28] Wagner, D. R., Reiser, K. M., and Lotz, J. C., 2006, "Glycation Increases Human Annulus Fibrosus Stiffness in Both Experimental Measurements and Theoretical Predictions," J. Biomech., 39, pp. 1021-1029.

[29] Spencer, A. J. M., 1984, Continuum Theory of the Mechanics of FibreReinforced Composites, Springer, New York.

[30] Klisch, S. M., and Lotz, J. C., 1999, "Application of a Fiber-Reinforced Continuum Theory to Multiple Deformations of the Annulus Fibrosus," J. Biomech., 32(10), pp. 1027-1036.

[31] Elliott, D. M., and Setton, L. A., 2000, "A Linear Material Model for FiberInduced Anisotropy of the Anulus Fibrosus,” J. Biomech. Eng., 122, pp. 173 179.

[32] Eberlein, R., Holzapfel, G. A., and Schulze-Bauer, C. A., 2001, "An Anisotropic Constitutive Model for Annulus Tissue and Enhanced Finite Element Analyses of Intact Lumbar Disc Bodies," Comput. Methods Biomech. Biomed. Eng., 4, pp. 209-230.

[33] Spencer, A. J. M., 1971, "Theory of Invariants," Continuum Physics, A. C. Eringen, ed., Academic, New York, pp. 240-353.

[34] Schroder, J., Neff, P., and Balzani, D., 2005, "A Variational Approach for Materially Stable Anisotropic Hyperelasticity," Int. J. Solids Struct., 42, pp. 4352-4371.

[35] Wagner, D. R., 2002, “A Mechanistic Strain Energy Function and Experimental Results for the Human Annulus Fibrosus," Ph.D. thesis, U. C. Berkeley, Berkeley, CA.

[36] Itskov, M., and Aksel, N., 2004, "A Class of Orthotropic and Transversely Isotropic Hyperelastic Constitutive Models Based on a Polyconvex Strain Energy Function,” Int. J. Solids Struct., 41, pp. 3833-3848.

[37] Chang, D. G., 1999, "Structure and Function Relationships of Articular Cartilage in Osteoarthritis," Ph.D. thesis, University of California, San Diego, La Jolla, CA.

[38] Jurvelin, J. S., Buschmann, M. D., and Hunziker, E. B., 1997, "Optical and 
Mechanical Determination of Poisson's Ratio of Adult Bovine Humeral Articular Cartilage,” J. Biomech., 30, pp. 235-241.

[39] Wong, M., Ponticiello, M., Kovanen, V., and Jurvelin, J. S., 2000, "Volumetric Changes of Articular Cartilage During Stress Relaxation in Unconfined Compression," J. Biomech., 33(9), pp. 1049-1054.

[40] Williamson, A. K., Chen, A. C., Masuda, K., Thonar, E. J.-M. A., and Sah, R. L., 2003, "Tensile Mechanical Properties of Bovine Articular Cartilage: Variations with Growth and Relationships to Collagen Network Components," J. Orthop. Res., 21, pp. 872-880.

[41] Broom, N. D., and Poole, C. A., 1983, "Articular Cartilage Collagen and Proteoglycans. Their Functional Interdependency," Arthritis Rheum., 26(9), pp. 1111-1119.

[42] Vynios, D. H., Papageorgakopoulou, N., Sazakli, H., and Tsiganos, C. P., 2001, "The Interactions of Cartilage Proteoglycans with Collagens are Determined by Their Structures," Biochimie, 83, pp. 899-906.

[43] Hendrickx, S., Thomas, P., Preston, B., and Stanton, P., 2001, "Partial Characterization of Matrix Components Interacting with Cartilage Proteoglycans," Arch. Biochem. Biophys., 390(2), pp. 186-194.

[44] Jeffery, A. K., Blunn, G. W., Archer, C. W., and Bentley, G., 1991, "ThreeDimensional Collagen Architecture in Bovine Articular Cartilage," J. Bone Jt. Surg., Br. Vol. 73-B, 795-801.

[45] Benninghoff, A., 1925, "Form und Bau der Gelenkknorpel in Ihren Beziehungen zur Funktion. Zweiter Teil: der Aufbau des Gelenkknorpels in Seinen Beziehungen zur Funktion," Z. Zellforsch Mikrosk Anat., 2, pp. 783-862.

[46] Lanir, Y., 1983, “Constitutive Equations for Fibrous Connective Tissues," J. Biomech., 16, pp. 1-12.

[47] Gasser, T. C., Ogden, R. W., and Holzapfel, G. A., 2006, "Hyperelastic Mod- elling of Arterial Layers with Distributed Collagen Fibre Orientations," J. R. Soc., Interface, 3, pp. 15-35.

[48] Anderson, A. F., Ellis, B. J., Maas, S. A., Peters, C. L., Ateshian, G. A., and Weiss, J. A., 2006, "Experimental Measurement and Finite Element Prediction of Cartilage Contact Pressures in the Human Hip," Proceedings ASME Summer Bioengineering Conference, Amelia Island, FL.

[49] Klisch, S. M., Sah, R. L., and Hoger, A., 2000, "A Growth Mixture Theory for Cartilage," Mechanics in Biology, J. Casey and G. Bao, eds., ASME, AMD242, New York, pp. 229-242.

[50] Klisch, S. M., Van Dyke, T., and Hoger, A., 2001, "A Theory of Volumetric Growth for Compressible Elastic Materials," Math. Mech. Solids, 6, pp. 551575 .

[51] Klisch, S. M., and Hoger, A., 2003, "Volumetric Growth of Thermoelastic Materials and Mixtures," Math. Mech. Solids, 8, pp. 377-402.

[52] Klisch, S. M., Chen, S. S., Sah, R. L., and Hoger, A., 2003, "A Growth Mixture Theory for Cartilage with Applications to Growth-Related Experiments on Cartilage Explants," J. Biomech. Eng., 125, pp. 169-179.

[53] Klisch, S. M., Sah, R. L., and Hoger, A., 2005, "A Cartilage Growth Mixture Model for Infinitesimal Strains: Solutions of Boundary-Value Problems Related to In Vitro Growth Experiments," Biomech. Model. Mechanobiol., 3(4), pp. 209-223.

[54] Davol, A., Bingham, M. S., Sah, R. L., and Klisch, S. M., 2007, "A Nonlinear Finite Element Model of Cartilage Growth,” Biomech. Model. Mechanobiol., in press.

[55] Smith, G. D., Knutsen, G., and Richardson, J. B., 2005, “A Clincial Review of Cartilage Repair Techniques," J. Bone Jt. Surg., Am. Vol. 87B(4), pp. 445449. 